\title{
Correlation of Net Atrioventricular Compliance with the Outcome of Successful Percutaneous Transvenous Mitral Valve Commissurotomy.
}

\author{
Rajib Ghosh, Abdullah Al Shafi Majumder, MM Hossain, Abdul Momen, M Aziz, Mohammad Ullah, \\ MZ Rahman, MA Rahman \\ Department of Cardiology, NICVD, Dhaka.
}

\begin{abstract}
:
Keywords:

Percutaneous

transvenous

mitral valve

commissurotomy,

Mitral stenosis,

Atrioventricular

compliance,

Rheumatic heart

disease.

Background: In patients with mitral stenosis the prognostic significance of net atrioventricular compliance has already been established. It was found that left atrial compliance significantly improve immediately after successful balloon mitral valvotomy. This study was conducted to evaluate the immediate improvement of atrioventricular compliance following percutaneous Transvenous mitral commissurotomy (PTMC) \& to see the relation of post PTMC atrioventricular compliance with immediate clinical \& other echocardiographic outcomes.

Methods: This prospective observational study was undertaken in the Department of Cardiology, National Institute of Cardiovascular diseases, Dhaka. A total of 50 patients who had had successful PTMC were selected. All patients were evaluated clinically and echocardiographically 24 hours before PTMC, immediately after PTMC and 72 hours after PTMC.

Results: Atrioventricular compliance improved significantly following PTMC (mean Cn $3.42 \pm$ $1.64 \mathrm{ml} / \mathrm{mm} \mathrm{Hg}$ vs. $6.38 \pm 2.81 \mathrm{ml} / \mathrm{mm} \mathrm{Hg}, \mathrm{p}<0.001$ ). After successful PTMC comparison of clinical \& echocardiographic parameters revealed mitral valve area, pulmonary arterial systolic pressure, transmitral peak and mean pressure gradient and NYHA class for dyspnoea had statistically significant difference among patients with atrioventricular compliance $>4 \mathrm{ml} / \mathrm{mm}$ of $\mathrm{Hg}$ and patients with atrioventricular compliance $d " 4 \mathrm{ml} / \mathrm{mm}$ of $\mathrm{Hg}$. NYHA class for heart failure, transmitral peak and mean pressure gradient, pulmonary artery systolic pressure were negatively correlated to atrioventricular compliance. Index left atrial anteroposterior diameter had no significant correlation with atrioventricular compliance.

Conclusion: Successful PTMC is associated with immediate significant improvement in atrioventricular compliance. Atrioventricular compliance is inversely correlated with NYHA functional status, transmitral pressure gradient \& pulmonary artery systolic pressure following successful PTMC. Post PTMC atrioventricular compliance is also positively correlated with pre PTMC atrioventricular compliance.
\end{abstract}

(Cardiovasc. j. 2016; 8(2): 99-109)

\section{Introduction:}

Rheumatic mitral valvular heart disease is a common and major cause of cardiovascular morbidity and mortality in Bangladesh. ${ }^{1}$ It is a chronic manifestation of rheumatic carditis, which may occur in $90 \%$ cases of rheumatic fever at 3 years of age to around $30 \%$ in adolescence. ${ }^{2}$ It is estimated that there are over 15 million cases of rheumatic heart disease (RHD) worldwide, with 282,000 new cases and 233,000 deaths annually. ${ }^{3}$

Several studies were on the prevalence of rheumatic heart disease reporting $0.14 / 1000$ in
Japan, 1.86/1000 in China, 0.5/1000 in Korea, 4.5/ 1000 in India. ${ }^{4}$ A community based study showed the prevalence of rheumatic heart disease is 1.3 per 1000 in rural Bangladesh. ${ }^{5}$

Mitral stenosis (MS) is the commonest (54\%) of all rheumatic heart diseases. ${ }^{6}$ The pathologic process of RHD produces fusion of the commissures, mitral valve calcification and fusion and shortening of chordae tendinae; the combination of these processes and their severity results in various grades of stenosis of the mitral valve. $^{7}$

Address of correspondence: Dr Rajib Ghosh, Department of Cardiology, National Institute of cardiovascular Diseases, Dhaka, Bangladesh. Email: rajib119@gmail.com 
The basic hemodynamic feature of MS is an elevation of left atrial (LA) pressure, resulting from obstruction to antegrade flow across the mitral valve. The extent of narrowing of mitral valve orifice determines the degree of elevation of LA pressure. However, a wide spectrum of LA pressures exists in patients with MS despite nearly similar mitral valve area. A linear relationship does not exist between the LA pressure and valve orifice or between LA pressure and pulmonary artery pressure. It is known that atrial and pulmonary compliances influence atrial and pulmonary hemodynamic. It is due to the complex interactions between mitral orifice area, LA compliance and pulmonary compliance that a linear relationship does not exist between mitral orifice size and LA and pulmonary artery pressures. ${ }^{8}$

The term compliance is used to describe how easily a chamber of the heart or the lumen of a blood vessel expands when it is filled with a volume of blood. Physically, compliance (C) is defined as the change in volume ( $\mathrm{AV})$ divided by the change in pressure (ÄP) $(\mathrm{C}=\ddot{\mathrm{AV}} / \ddot{\mathrm{AP}}) .^{9}$

The net atrioventricular compliance (Cn) implies compliance characteristics of both the chambers atrium and ventricle, as a single unit. In case of pure mitral stenosis, left ventricular compliance is assumed to be normal. Therefore an abnormal Cn reflects abnormality of the atrial compliance. But it has been found that about one third of patients with mitral stenosis have reduced left ventricular compliance. ${ }^{10}$

Net $\mathrm{Cn}$ is an important determinant of pulmonary artery pressure in mitral stenosis independently of mitral valve (MV) area and transvalvular pressure gradients. Patients with low Cn were more symptomatic with symptoms corresponding to severe increases in pulmonary artery pressures during stress echocardiography. ${ }^{11}$

Mitral stenosis is best described as a disease continuum, with no single value that can define its severity. More specifically, a wide variation in pulmonary pressures for the same range of mitral obstruction emphasizes the need to identify key factors implicated in the development of pulmonary hypertension and functional limitation in MS. ${ }^{12}$

The presence of pulmonary hypertension is fundamental in the clinical decision-making process for mitral stenosis (MS). ${ }^{13,14}$ It strongly expresses valve disease severity and conveys adverse effects on functional status, exercise tolerance, and prognosis. ${ }^{15,16}$ However, pulmonary pressure may not be uniquely determined by the stenotic lesion itself but by a combination of hemodynamic parameters. ${ }^{17,18}$ Several factors may contribute to clinical presentation and outcome in MS. Leftheart compliance plays a crucial role in the occurrence of pulmonary hypertension and symptoms. ${ }^{19}$

Li et $\mathrm{al}^{20}$ found that $\mathrm{Cn}$ was the only Doppler echocardiographic variable that independently predicted pulmonary artery pressure. Cn can be readily calculated by Doppler echocardiography.

Flachskampf et $\mathrm{al}^{21}$ presented analytic and numeric evidence supporting the quantitative assessment of $\mathrm{Cn}$ from transmitral velocity profiles, deriving a simple equation that relates it to effective MV area and E-wave down slope. This equation has been validated in vitro and accurately predicts Cn. Subsequently Schwammenthal et al ${ }^{22}$ showed that $\mathrm{Cn}$ can be calculated noninvasively and reproducibly in the clinical setting and correlates well with invasively determined values.

Nunes et $a^{23}$ showed that net atrioventricular compliance, $\mathrm{Cn} \leq 4 \mathrm{~mL} / \mathrm{mm} \mathrm{Hg}$ best predicted unfavorable outcome in patients with mitral stenosis and $\mathrm{Cn}$ was found as a powerful predictor of adverse outcome, adding prognostic information beyond that provided by clinical evaluation and MV area.

In another study it was showed that $\mathrm{Cn}$ is an independent predictor of death in patients with significant MS who underwent percutaneous mitral valvuloplasty (PMV), even after adjustment for important prognostic factors like demographics, clinical factors (including co morbidity index), pulmonary artery pressure, ventricular function, degree of mitral regurgitation (MR), and immediate procedural results (MR severity and mitral valve area after PMV). ${ }^{24}$

Kapoor et $\mathrm{al}^{25}$ found that left atrial compliance significantly improved immediately after successful balloon mitral valvotomy, irrespective of the pre valvotomy left atrial pressure.

The effect of a successful PTMC on net atrioventricular compliance $(\mathrm{Cn})$ is still not 
evaluated. The relation of post PTMC net atrioventricular compliance $(\mathrm{Cn})$ and outcome parameters of PTMC has not been tested. As post PTMC atrial compliance increases, so we have hypothesized that post PTMC net atrioventricular compliance $(\mathrm{Cn})$ will also be increased and it will be correlated with outcome parameters of PTMC, both clinical and echocardiographic.

\section{Methods:}

This prospective observational study was conducted in the Department of Cardiology, NICVD, from June, 2014 to May, 2015. A total of 50 patients who had had planimetered mitral valve area $<1.5$ $\mathrm{cm}^{2}$, Wilkin's score $\leq 10$, isolated mitral stenosis or with not more than grade I mitral regurgitation and successful PTMC (After PTMC MVA $\geq 1.5 \mathrm{~cm}^{2}$ or $\geq 50 \%$ increased from baseline, mean trans valvular pressure gradient $<5 \mathrm{~mm} \mathrm{Hg}$ or $\geq 50 \%$ reduced from baseline) were selected. Patients with concomitant pregnancy, hypertension and documented coronary artery disease were excluded. The study protocol was approved by the Ethical Review Committee of NICVD.

A complete clinical evaluation was performed on all patients the day before PTMC, immediately after PTMC and 72 hours after PTMC. Functional status was determined using the New York Heart Association (NYHA) classification based on functional capacity and dyspnea. Transthoracic echocardiography was done the day before PTMC, immediately after PTMC for procedural success and complications and 72 hours after PTMC by one expert. Patients were evaluated by 2D, Mmode and Doppler echocardiography, imaging was performed and analyzed according to the recommendations of the American Society of Echocardiography using a commercially available echocardiography (GE Vivid 5). Standard echocardiographic parameters were averaged from 3 consecutive cardiac cycles for patients in sinus rhythm or 5 consecutive cycles for patients in atrial fibrillation. Mitral valve area was measured by planimetry in parasternal short axis view. MV morphology was evaluated using the Wilkins score. Peak and mean transmitral diastolic pressure gradients were measured from Doppler profiles recorded in the apical 4-chamber view. Systolic pulmonary artery pressure (SPAP) was measured by continuous-wave Doppler tricuspid regurgitant velocity using the simplified Bernoulli equation. Left atrial diameter, left ventricular end diastolic diameter, left ventricular end systolic diameter and LV ejection fraction were measured by $2 \mathrm{D}$ and $\mathrm{M}$ mode study. Procedural complications were observed immediately after PTMC. Hemodynamic was recorded by measuring pulmonary artery systolic pressure, aortic pressure gradient, transmitral pressure gradient (peak) and LV ejection fraction. Invasive measurement of change in aortic \& LA pressure after PTMC was recorded.

\section{Assessment of Cn}

Cn was determined noninvasively by means of Doppler echocardiography, $\mathrm{Cn}(\mathrm{mL} / \mathrm{mm} \mathrm{Hg})=1270$ $\times$ (planimetric MV area [cm2]/E-wave down slope $\left.\left[\mathrm{cm} / \mathrm{s}^{2}\right]\right) .{ }^{21}$ In those patients with nonlinear diastolic flow, the mid-diastolic flow was used, which was felt to best represent the valve stenosis as opposed to early LA depressurization, and the slope was extrapolated back to obtain the initial maximal velocity.

\section{Statistical Methods:}

The data obtained from the study were analyzed and significance of differences was estimated by using statistical methods. Data was expressed as mean \pm SD. The SPSS Statistical Software (17.0 version, SPSS Inc., Chicago, Illinois, USA) was used for data analysis. Continuous variables were expressed in mean \& standard deviation and categorical variables as frequency and percentage. Paired Student's t-test was used to compare normally distributed continuous variables of two groups that are dependent on each other. Again two sample unpaired t-test was used to compare normally distributed continuous variables of two groups that are not dependent on each other. $\mathrm{x}^{2}$ test was used to compare two groups of unpaired discrete variables. To show the relationship between quantitative variables Pearson correlation test \& in case of one quantitative and one ordinal variable Spearman's rank test were performed. A p-value $<0.05$ was considered statistically significant.

\section{Results:}

Mean age of patients of PTMC was 33.72 years ( $\mathrm{SD} \pm 8.71$ years). Age varied from 20 to 50 years. 78 percent of the patients were female and 22 percent was male. (Fig 1). Baseline clinical 


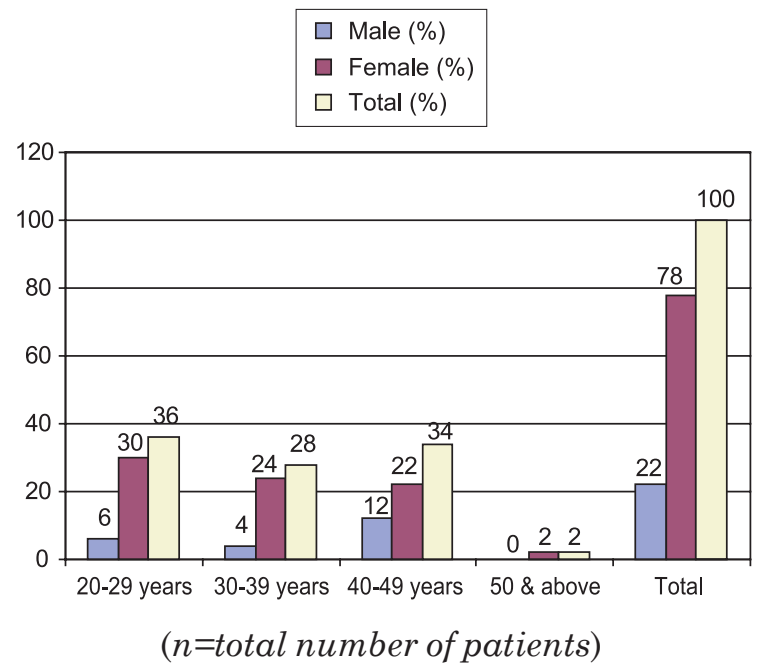

Fig 1: Age and sex distribution of patients of PTMC

evaluation revealed that only 9 patients (18\%) had history suggestive of rheumatic fever and none of them took prophylaxis against rheumatic fever. 5 patients (10\%) had atrial fibrillation. 11 patients (22\%) had history of left atrial spontaneous echo contrast or left atrial thrombus or previous ischemic stroke. 2 patients (4\%) had history of previous mitral valve intervention (CMC or PTMC). All patients ( $n=50,100 \%)$ had shortness of breath, other symptoms were palpitation (50\%), chest pain, cough, orthopnoea, PND \& haemoptysis (Table II).

Baseline echocardiographic evaluation revealed most of the patient had very severe mitral stenosis (mean MVA was $0.80 \pm 0.17 \mathrm{~cm}^{2}$, mean trans mitral peak pressure gradient was $28.83 \pm 9.39 \mathrm{~mm} \mathrm{Hg}$, mean trans mitral mean pressure gradient was $16.54 \pm 6.17 \mathrm{~mm} \mathrm{Hg}$ ) with severely elevated pulmonary artery systolic pressure (mean pulmonary artery systolic pressure was $73.28 \pm$ $29.43 \mathrm{~mm} \mathrm{Hg}$ ). (Table-II).

Table I

Distribution of patients by baseline clinical characteristics $(n=50)$.

\begin{tabular}{|c|c|c|c|}
\hline \multicolumn{2}{|l|}{ Clinical characteristics } & Frequency & Percentage \\
\hline \multicolumn{2}{|c|}{ History suggestive of rheumatic fever } & 09 & 18 \\
\hline \multirow{2}{*}{\multicolumn{2}{|c|}{$\begin{array}{l}\text { Atrial fibrillation } \\
\text { History of left atrial spontaneous echo contrast or left atrial } \\
\text { thrombus or ischemic stroke }\end{array}$}} & 05 & 10 \\
\hline & & 11 & 22 \\
\hline \multirow{8}{*}{$\begin{array}{l}\text { History of mitral valve } \\
\text { Clinical presentations }\end{array}$} & tervention (PTMC/CMC) & 02 & 4 \\
\hline & Shortness of breath & 50 & 100 \\
\hline & Palpitation & 25 & 50 \\
\hline & Chest pain & 18 & 36 \\
\hline & Cough & 17 & 34 \\
\hline & Haemoptysis & 04 & 08 \\
\hline & Orthopnoea & 11 & 22 \\
\hline & Paroxysmal nocturnal dyspnoea (PND) & 08 & 16 \\
\hline
\end{tabular}

$\mathrm{n}=$ total number of patients

Table-II

Distribution of patients by baseline clinical echocardiographic findings $(n=50)$.

\begin{tabular}{lcc}
\hline Clinical examination \& echocardiographic findings & Mean & Standard deviation \\
\hline Left ventricular end diastolic diameter $(\mathrm{mm})$ & 39 & 5.27 \\
Left ventricular ejection fraction $(\%)$ & 65.38 & 6.10 \\
Mitral valve area (MVA) $\left(\mathrm{cm}^{2}\right)$ & 0.80 & 0.17 \\
Index left atrial antero posterior diameter $\left(\mathrm{mm} / \mathrm{m}^{2}\right)$ & 32.11 & 6.15 \\
Trans mitral peak pressure gradient $(\mathrm{mm} \mathrm{Hg})$ & 28.83 & 9.39 \\
Trans mitral mean pressure gradient $(\mathrm{mm} \mathrm{Hg})$ & 16.54 & 6.17 \\
Pulmonary artery systolic pressure $(\mathrm{mm} \mathrm{Hg})$ & 73.28 & 29.43 \\
Net atrioventricular compliance, $\mathrm{Cn}(\mathrm{ml} / \mathrm{mmHg})$ & 3.42 & 1.64 \\
\hline
\end{tabular}

$\mathrm{n}=$ total number of patients 
All patients (32\%) presented with NYHA class II heart failure improved to NYHA class I after PTMC. But those $42 \%$ patients who presented with NYHA class IV heart failure, $14 \%$ improved to NYHA class I and $28 \%$ still remained symptomatic (22\% NYHA class II and 6\% NYHA class III) after PTMC.(Table-III).

Following PTMC pulmonary arterial systolic pressure, index left atrial anteroposterior diameter, transmitral peak and mean pressure gradient, invasively measured mean left atrial pressure were significantly reduced. On the other hand, mitral valve area significantly increased after PTMC (Table IV). After PTMC Net atrioventricular compliance $(\mathrm{Cn})$ significantly increased compared to baseline net atrioventricular compliance $(3.42$ $\pm 1.64 \mathrm{ml} / \mathrm{mm} \mathrm{Hg}$ vs. $6.38 \pm 2.81 \mathrm{ml} / \mathrm{mm} \mathrm{Hg}, \mathrm{p}<$ 0.001).Comparison of outcome among patients with
$\mathrm{Cn}>4 \mathrm{ml} / \mathrm{mm}$ of $\mathrm{Hg}$ and patients with $\mathrm{Cn} \leq 4 \mathrm{ml} /$ $\mathrm{mm}$ of $\mathrm{Hg}$ following PTMC revealed Mitral valve area, Pulmonary arterial systolic pressure, transmitral peak and mean pressure gradient had statistically significant difference. On the other hand index left atrial anteroposterior diameter and invasively measured mean left atrial pressure had no statistically significant difference (Table-V).

NYHA class for dyspnoea showed statistically significant difference among patients with $\mathrm{Cn}>4$ $\mathrm{ml} / \mathrm{mm}$ of $\mathrm{Hg}$ and patients with $\mathrm{Cn} \leq 4 \mathrm{ml} / \mathrm{mm}$ of Hg following PTMC $(p<0.001)$ (Table VI).

Again, patients who gained MVA $\geq 1.5 \mathrm{~cm}^{2}$ following PTMC, comparison of outcome among them with $\mathrm{Cn}>4 \mathrm{ml} / \mathrm{mm}$ of $\mathrm{Hg}$ and $\mathrm{Cn} \leq 4 \mathrm{ml} / \mathrm{mm}$ of $\mathrm{Hg}$ revealed Pulmonary arterial systolic pressure, transmitral peak and mean pressure gradient \& Cn had statistically significant difference. On the other hand

Table-III

Distribution of patients by NYHA functional class before \& after successful PTMC (Cross Tabulation)

\begin{tabular}{llcccc}
\hline & & After PTMC (\%) & \multicolumn{2}{c}{ Total (\%) } \\
& & NYHA & $\begin{array}{c}\text { NYHA } \\
\text { Class II }\end{array}$ & $\begin{array}{c}\text { NYHA } \\
\text { Class III }\end{array}$ & \\
\hline Before PTMC (\%) & NYHA Class II & 32 & 0 & 0 & 32 \\
& NYHA Class III & 12 & 14 & 0 & 26 \\
& NYHA Class IV & 14 & 22 & 6 & 42 \\
\hline Total $(\%)$ & 58 & 36 & 6 & 100 & \\
\hline
\end{tabular}

Table IV

Comparison of echocardiographic \& invasive findings before and after successful PTMC $(n=50)$.

\begin{tabular}{lccc}
\hline Echocardiographic and invasive findings & \multicolumn{2}{c}{ Groups } & p value \\
\cline { 2 - 3 } & Before PTMC & After PTMC & \\
\hline Mitral valve area $\left(\mathrm{cm}^{2}\right)$ & $0.80 \pm 0.17$ & $1.47 \pm 0.27$ & $<0.001$ \\
Index left atrial antero posterior diameter $\left(\mathrm{mm} / \mathrm{m}^{2}\right)$ & $32.11 \pm 6.15$ & $28.52 \pm 4.37$ & $<0.001$ \\
Trans mitral peak pressure gradient $(\mathrm{mm} \mathrm{Hg})$ & $28.83 \pm 9.39$ & $13.46 \pm 5.36$ & $<0.001$ \\
Trans mitral mean pressure gradient $(\mathrm{mm} \mathrm{Hg})$ & $16.54 \pm 6.17$ & $6.93 \pm 3.68$ & $<0.001$ \\
Pulmonary artery systolic pressure $(\mathrm{mm} \mathrm{Hg})$ & $73.28 \pm 29.43$ & $48.79 \pm 21.58$ & $<0.001$ \\
Invasively measured mean left atrial pressure $(\mathrm{mm} \mathrm{Hg})$ & $28.53 \pm 10.02$ & $15.12 \pm 7.36$ & $<0.001$ \\
\hline
\end{tabular}


MVA, index left atrial anteroposterior diameter and invasively measured mean left atrial pressure had no statistically significant difference (Table VII). NYHA class for dyspnoea showed statistically significant difference among patients with $\mathrm{Cn}>4 \mathrm{ml}$ $\mathrm{mm}$ of $\mathrm{Hg}$ and patients with $\mathrm{Cn} \leq 4 \mathrm{ml} / \mathrm{mm}$ of $\mathrm{Hg}$ following PTMC ( $p<0.001)$ (Table VIII).

Comparison of outcome following successful PTMC among patients with MVA $<1.5 \mathrm{~cm}^{2}$ and patients with MVA $\geq 1.5 \mathrm{~cm}^{2}$ revealed mitral valve area and net atrioventricular compliance had statistically significant difference but pulmonary arterial systolic pressure, transmitral peak and mean pressure gradient, index left atrial anteroposterior diameter and invasively measured mean left atrial pressure had no statistically significant difference among patients of these two groups. (Table IX)

Table-V

Comparison of echocardiographic \& invasive findings among patients with $\mathrm{Cn}>4 \mathrm{ml} / \mathrm{mm}$ of $\mathrm{Hg}$ and patients with $\mathrm{Cn} \leq 4 \mathrm{ml} / \mathrm{mm}$ of $\mathrm{Hg}$ after successful PTMC. $(n=50)$.

Echocardiographic and invasive findings

Mitral valve area $\left(\mathrm{cm}^{2}\right)$

Net atrioventricular compliance $(\mathrm{ml} / \mathrm{mm} \mathrm{Hg})$

Index left atrial antero posterior diameter $\left(\mathrm{mm} / \mathrm{m}^{2}\right)$

Trans mitral peak pressure gradient $(\mathrm{mm} \mathrm{Hg})$

Trans mitral mean pressure gradient $(\mathrm{mm} \mathrm{Hg})$

Pulmonary artery systolic pressure $(\mathrm{mm} \mathrm{Hg})$

Invasively measured mean left atrial pressure ( $\mathrm{mm} \mathrm{Hg}$ )
Groups after PTMC

$\mathrm{Cn}>4 \mathrm{ml} /$

$\mathrm{mm}$ of $\mathrm{Hg}$

$(\mathrm{n}=38)$

$1.56 \pm 0.22$

$7.4 \pm 2.4$

$28.11 \pm 4.59$

$11.77 \pm 3.80$

$5.65 \pm 2.26$

$42.20 \pm 15.49$

$13.84 \pm 5.90$
$\mathrm{Cn} \leq 4 \mathrm{ml} /$

p value

$\mathrm{mm}$ of $\mathrm{Hg}$

$(\mathrm{n}=12)$

$1.24 \pm 0.28 \quad 0.002$

$3.1 \pm 0.49 \quad<0.001$

$29.84 \pm 3.40$

$18.80 \pm 6.20$

$10.98 \pm 4.42$

$69.64 \pm 25.37$

$16.75 \pm 9.49$

0.172

0.002

0.002

0.003

0.334

Table-VI

Comparison of NYHA class for dyspnoea among patients with $\mathrm{Cn} \leq 4 \mathrm{ml} / \mathrm{mm}$ of $\mathrm{Hg}$ and patients with $\mathrm{Cn}>4 \mathrm{ml} / \mathrm{mm}$ of $\mathrm{Hg}$ after successful PTMC $(n=50)$.

\begin{tabular}{lccccc}
\hline & $\begin{array}{c}\text { NYHA class I } \\
\text { frequency) }\end{array}$ & $\begin{array}{c}\text { (NYHA class II } \\
\text { (frequency) }\end{array}$ & $\begin{array}{c}\text { NYHA class III } \\
\text { (frequency) }\end{array}$ & Row total & P value \\
\hline $\mathrm{Cn} \leq 4 \mathrm{ml} / \mathrm{mm}$ of $\mathrm{Hg}$ & 01 & 08 & 03 & 12 & $<0.001$ \\
$\mathrm{Cn}>4 \mathrm{ml} / \mathrm{mm}$ of $\mathrm{Hg}$ & 28 & 10 & 0 & 38 & \\
Column total & 29 & 18 & 3 & Grand total $=50$ & \\
\hline
\end{tabular}

Table-VII

Comparison of echocardiographic \& invasive findings among patients with $M V A \geq 1.5 \mathrm{~cm}^{2}$ after successful PTMC $(n=35)$.

\begin{tabular}{lccc}
\hline Echocardiographic and invasive findings & \multicolumn{2}{c}{ Groups after PTMC } & p value \\
\cline { 2 - 3 } & $\begin{array}{c}\mathrm{Cn}>4 \mathrm{ml} / \mathrm{mm} \\
\text { of } \mathrm{Hg}(\mathrm{n}=30)\end{array}$ & $\begin{array}{c}\mathrm{Cn} \leq 4 \mathrm{ml} / \mathrm{mm} \\
\text { of } \mathrm{Hg}(\mathrm{n}=5)\end{array}$ & \\
\hline Mitral valve area $\left(\mathrm{cm}^{2}\right)$ & $1.63 \pm 0.17$ & $1.52 \pm 0.16$ & 0.174 \\
Net atrioventricular compliance $(\mathrm{ml} / \mathrm{mm} \mathrm{Hg})$ & $7.59 \pm 2.59$ & $3.26 \pm 0.31$ & 0.001 \\
Index left atrial antero posterior diameter $\left(\mathrm{mm} / \mathrm{m}^{2}\right)$ & $28.00 \pm 5.01$ & $28.39 \pm 2.21$ & 0.866 \\
Trans mitral peak pressure gradient $(\mathrm{mm} \mathrm{Hg})$ & $11.33 \pm 3.72$ & $20.40 \pm 6.06$ & $<0.001$ \\
Trans mitral mean pressure gradient $(\mathrm{mm} \mathrm{Hg})$ & $5.42 \pm 2.14$ & $11.40 \pm 4.77$ & $<0.001$ \\
Pulmonary artery systolic pressure $(\mathrm{mm} \mathrm{Hg})$ & $41.39 \pm 15.47$ & $68.00 \pm 31.36$ & 0.005 \\
Invasively measured mean left atrial pressure $(\mathrm{mm} \mathrm{Hg})$ & $14.40 \pm 3.72$ & $18.00 \pm 6.78$ & 0.247 \\
\hline
\end{tabular}


Table-VIII

Comparison of NYHA class for dyspnoea among patients with $M V A \geq 1.5 \mathrm{~cm}^{2}$ after successful PTMC. (N=35)

\begin{tabular}{lcccc}
\hline & $\begin{array}{c}\text { NYHA class I } \\
\text { (frequency) }\end{array}$ & $\begin{array}{c}\text { NYHA class II } \\
\text { (frequency) }\end{array}$ & Row total & p value \\
\hline $\mathrm{Cn} \leq 4 \mathrm{ml} / \mathrm{mm}$ of $\mathrm{Hg}$ & 01 & 04 & 05 & $<0.001$ \\
$\mathrm{Cn}>4 \mathrm{ml} / \mathrm{mm}$ of $\mathrm{Hg}$ & 23 & 07 & 30 & \\
Column total & 24 & 11 & Grand total $=35$ & \\
\hline
\end{tabular}

Table-IX

Comparison of echocardiographic \& invasive findings among patients with $M V A \geq 1.5 \mathrm{~cm}^{2}$ and patients with $M V A<1.5 \mathrm{~cm}^{2}$ after successful PTMC. $(n=50)$

\begin{tabular}{lccc}
\hline Echocardiographic and invasive findings & \multicolumn{2}{c}{ Groups after PTMC } & p value \\
\cline { 2 - 3 } & $\begin{array}{c}\left.\text { MVA } \geq 1.5 \mathrm{~cm}^{2}\right) \\
(\mathrm{n}=35)\end{array}$ & $\begin{array}{c}\text { MVA }<1.5 \mathrm{~cm}^{2} \\
(\mathrm{n}=15)\end{array}$ & \\
\hline Mitral valve area $\left(\mathrm{cm}^{2}\right)$ & $1.62 \pm 0.18$ & $1.15 \pm 0.14$ & $<0.001$ \\
Net atrioventricular compliance $(\mathrm{ml} / \mathrm{mm} \mathrm{Hg})$ & $6.97 \pm 2.85$ & $5.00 \pm 2.24$ & 0.02 \\
Index left atrial antero posterior diameter $\left(\mathrm{mm} / \mathrm{m}^{2}\right)$ & $28.06 \pm 4.70$ & $29.61 \pm 3.40$ & 0.25 \\
Trans mitral peak pressure gradient $(\mathrm{mm} \mathrm{Hg})$ & $12.63 \pm 5.15$ & $15.42 \pm 5.50$ & 0.09 \\
Trans mitral mean pressure gradient $(\mathrm{mm} \mathrm{Hg})$ & $6.28 \pm 3.33$ & $8.45 \pm 4.13$ & 0.056 \\
Pulmonary artery systolic pressure $(\mathrm{mm} \mathrm{Hg})$ & $45.20 \pm 20.23$ & $57.20 \pm 25.00$ & 0.07 \\
Invasively measured mean left atrial pressure $(\mathrm{mm} \mathrm{Hg})$ & $14.91 \pm 6.35$ & $13.67 \pm 8.33$ & 0.56 \\
\hline
\end{tabular}

Table-X

Correlation of different echocardiographic parameters and invasively measured mean left atrial pressure with net atrioventricular compliance (Cn) following successful PTMC. $(n=50)$

Correlated variables

Independent (X)

Net atrioventricular compliance $(\mathrm{Cn})$

Net atrioventricular compliance (Cn)

Net atrioventricular compliance (Cn)

Net atrioventricular compliance (Cn)

Net atrioventricular compliance (Cn)

Net atrioventricular compliance $(\mathrm{Cn})$

Dependent (Y)

\begin{tabular}{lcc} 
Mitral valve area & 0.452 & 0.001 \\
Trans-mitral peak pressure gradient & -0.613 & $<0.001$ \\
Trans-mitral mean pressure gradient & -0.615 & $<0.001$ \\
Pulmonary artery systolic pressure & -0.567 & $<0.001$ \\
Index left atrial antero posterior diameter & -0.034 & 0.815 \\
Invasively measured mean left atrial pressure & -0.334 & 0.018 \\
\hline
\end{tabular}

Correlation p-value co-efficient (r)

Echocardiographic parameters following PTMC showed that mitral valve area was positively correlated to net atrioventricular compliance (Cn). Transmitral peak pressure gradient, trans mitral mean pressure gradient and pulmonary artery systolic pressure were negatively correlated to net atrioventricular compliance (Cn), Index left atrial anteroposterior diameter had no significant correlation with net atrioventricular compliance (Cn). Again invasively measured mean left atrial pressure was found negatively correlated with net atrioventricular compliance (Cn). (Table X and Fig. - 3, 4). The pre PTMC net atrioventricular compliance was found to be positively correlated to post PTMC net atrioventricular compliance $(\mathrm{r}=$ 0.678, p < 0.001) (Table XI and Fig.-2). The post 


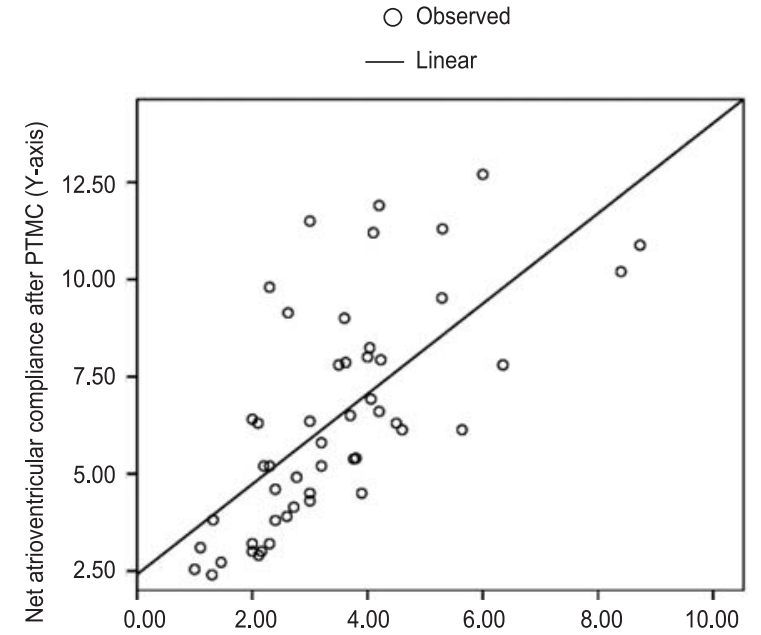

Fig.-2: Scatter plot showing correlation between net atrioventricular compliance before PTMC (Xaxis) and net atrioventricular compliance after successful PTMC (Y-axis). There is a nonlinear positive relationship between $C n$ before and Cn after PTMC.

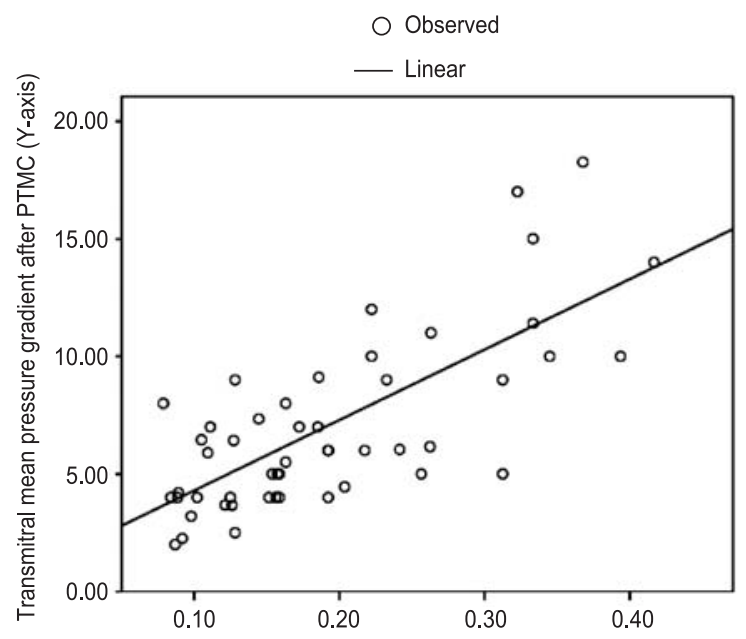

Fig.-3: Scatter plot showing correlation between transmitral mean pressure gradient ( $Y$-axis) and net atrioventricular stiffness (1/net atrioventricular compliance [Cn]) (X-axis) following successful PTMC. There is a nonlinear negative relationship between transmitral mean pressure gradient and Cn and a positive correlation between transmitral mean pressure gradient and net atrioventricular stiffness following successful PTMC.

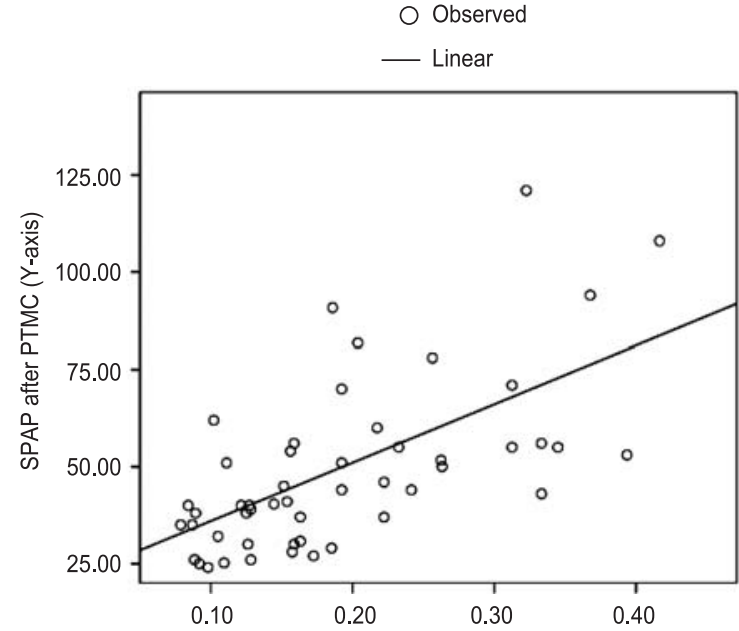

Fig.-4: Scatter plot showing correlation between systolic pulmonary artery pressure [SPAP] (Y-axis) and net atrioventricular stiffness (1/net atrioventricular compliance [Cn]) (X-axis) following successful PTMC. There is a nonlinear negative relationship between Cn and SPAP and a positive correlation between SPAP and net atrioventricular stiffness following successful PTMC.

PTMC NYHA class for heart failure was found to be negatively correlated to post PTMC net atrioventricular compliance $(r=-0.712, p<0.001)$ (Table XII).

In this study the mean age of the patients was $33.72 \pm 8.71$ years. From other studies of this institute, mean age of patients undergoing PTMC was found 29.7 7.7 years by Mannan, ${ }^{25} 28.2 \pm 6.7$ years by Kundu ${ }^{27}$ and $28.83 \pm 9.33$ years by Chowdhury. ${ }^{28}$ So, mean age of patients of this study was a bit higher than that found from other previous studies in the same institute. A comprehensive study by Hasan- Ali et al showed mean age $30 \pm 9$ years among patients undergoing PTMC. ${ }^{29}$

Functional class status evaluation revealed 32\% of study population was NYHA class II, $26 \%$ was in class III and $42 \%$ was in class IV. Mannan, ${ }^{26}$ in his study found $5 \%$ of this study population was in NYHA class I, $63.3 \%$ was in class II, $26.7 \%$ was in class III and $5 \%$ was in class IV. Severe symptomatic patients were more in our study group. Following PTMC all patients showed improvement regarding functional class. 
Mitral regurgitation was a frequent result of PTMC. Significant mitral regurgitation occurs in $3.3-10.5 \%$ of cases. ${ }^{30}$ In this study $24 \%$ patients developed moderate MR, 14\% patients developed mild MR. In this study MVA increased after PTMC from $0.80 \pm 0.17 \mathrm{~cm}^{2}$ to $1.47 \pm 0.27 \mathrm{~cm}^{2}$ by planimetry $(\mathrm{p}<0.001)$. Mannan ${ }^{26}$, in his study found MVA increase from $0.8 \pm 0.1 \mathrm{~cm}^{2}$ to $1.7 \pm 0.2 \mathrm{~cm}^{2}$ by planimetry. Study conducted by Kundu et $\mathrm{al}^{27}$ revealed increased MVA from $0.8 \pm 0.1 \mathrm{~cm}^{2}$ to $2.0 \pm 0.2 \mathrm{~cm}^{2}$ by planimetry. Kang et $\mathrm{al}^{31}$ showed MVA increased from $0.9 \pm 0.2 \mathrm{~cm}^{2}$ to $1.8 \pm 0.3 \mathrm{~cm}^{2}$. A study, done by Illius ${ }^{32}$ showed MVA increased from $0.76 \pm 0.2 \mathrm{~cm}^{2}$ to $1.8 \pm 0.3 \mathrm{~cm}^{2}$ by planimetry after PTMC which is also consistent with our present study.

In this study PPG was reduced from 28.83 \pm 9.39 $\mathrm{mm}$ of $\mathrm{Hg}$ to $13.46 \pm 5.36 \mathrm{~mm}$ of $\mathrm{Hg}(\mathrm{p}<0.001)$. In a study Mannan ${ }^{26}$, found reduction of PPG $24 \pm 7.2$ $\mathrm{mm}$ of $\mathrm{Hg}$ to $10.4 \pm 3.4 \mathrm{~mm}$ of $\mathrm{Hg}$. A study done by Kundu et $\mathrm{al}^{27}$ showed PPG reduced from 26.0 23.8 to $8.0 \pm 1.5 \mathrm{~mm}$ of $\mathrm{Hg}$. Illius ${ }^{32}$ showed decrease of PPG from $27.02 \pm 7.3$ to $12.01 \pm 5.3 \mathrm{~mm}$ of $\mathrm{Hg}$. Rahman ${ }^{33}$ showed decrease of PPG from $26.73 \pm 7.30$ to $11.79 \pm 5.11 \mathrm{~mm}$ of $\mathrm{Hg}$ after PTMC. So above mentioned studies are consistent with our present study.

In our study index left atrial anteroposterior diameter was reduced from $32.11 \pm 6.15 \mathrm{~mm} / \mathrm{m}^{2}$ to $28.52 \pm 4.37 \mathrm{~mm} / \mathrm{m}^{2}$. Mannan ${ }^{25}$, in his study found left atrial diameter was reduced from $4.3 \pm 0.6$ to $3.8 \pm 0.6 \mathrm{~cm}$ after PTMC. Illius ${ }^{31}$ has shown reduction of left atrial diameter from $4.8 \pm 0.7$ to $4.3 \pm 0.65 \mathrm{~cm}$ after PTMC. A study by Rahman ${ }^{33}$ showed reduction of left atrial diameter from $4.6 \pm 0.6$ to $4.3 \pm 0.7 \mathrm{~cm}$ after PTMC. So our study is comparable to above mentioned studies in respect of reduction of left atrial diameter after PTMC.

In this study pulmonary artery systolic pressure reduced from $73.28 \pm 29.43$ to $48.79 \pm 21.58 \mathrm{~mm}$ of $\mathrm{Hg}(\mathrm{p}<0.001)$. Post PTMC pulmonary artery systolic pressure, though found reduced significantly, still higher than that found in other studies. ${ }^{26,27}$

In this study net atrioventricular compliance increased from $3.42 \pm 1.64$ to $6.38 \pm 7.36 \mathrm{ml} / \mathrm{mm}$ of $\mathrm{Hg}$. Aditya Kapoor et al ${ }^{25}$ found that left atrial compliance significantly improved immediately after successful balloon mitral valvotomy, irrespective of the pre valvotomy left atrial pressure. Currently there is no available data showing the effect of a successful PTMC on net atrioventricular compliance.

In our study we found significant difference in trans mitral peak and mean pressure gradients and PASP among patients with $\mathrm{Cn}>4 \mathrm{ml} / \mathrm{mm} \mathrm{Hg} \& \mathrm{Cn} \leq 4$ $\mathrm{mi} / \mathrm{mm} \mathrm{Hg}$ following successful PTMC. Again the differences in index left atrial anteroposterior diameter and invasively measured mean left atrial pressure among these two groups were not statistically significant.

Comparison of NYHA class showed significant improvement of breathlessness in the group with $\mathrm{Cn}>4 \mathrm{ml} / \mathrm{mm}$ of $\mathrm{Hg}$ compared to the group with $\mathrm{Cn} \leq 4 \mathrm{ml} / \mathrm{mm}$ of $\mathrm{Hg}$. $(\mathrm{p}<0.001)$.

When the above comparisons done among the patients who had mitral valve area $\geq 1.5 \mathrm{~cm}^{2}$ following successful PTMC, then Pulmonary arterial systolic pressure, transmitral peak and mean pressure gradient, net atrioventricular compliance (Cn) and NYHA class for dyspnoea had statistically significant difference among patients with $\mathrm{Cn}>4 \mathrm{ml} / \mathrm{mm}$ of $\mathrm{Hg}$ and patients with $\mathrm{Cn}$ $\leq 4 \mathrm{ml} / \mathrm{mm}$ of $\mathrm{Hg}$ following successful PTMC. On the other hand MVA, index left atrial anteroposterior diameter and invasively measured mean left atrial pressure had no statistically significant difference among patients of those two groups following successful PTMC. So, patients' symptoms, PASP and trans mitral gradient independently related to post PTMC net atrioventricular compliance $(\mathrm{Cn})$.

In our study echocardiographic parameters with net atrioventricular compliance $(\mathrm{Cn})$ following PTMC showed that transmitral peak pressure gradient, trans mitral mean pressure gradient and pulmonary artery systolic pressure were negatively correlated to net atrioventricular compliance (Cn), Index left atrial anteroposterior diameter had no significant correlation with net atrioventricular compliance $(\mathrm{Cn}) \quad(\mathrm{r}=-0.613, \mathrm{p}<0.001$ and $\mathrm{r}=$ $0.615, \mathrm{p}<0.001$ and $\mathrm{r}=-0.567, \mathrm{p}<0.001$ and $\mathrm{r}=$ $0.334, p=0.815$ respectively). Again invasively measured mean left atrial pressure was found negatively correlated with net atrioventricular compliance $(\mathrm{Cn})(\mathrm{r}=-0.334, \mathrm{p}<0.001)$.

The pre PTMC net atrioventricular compliance was found to be positively correlated to post PTMC net 
atrioventricular compliance $(\mathrm{r}=0.678, \mathrm{p}<0.001)$. The post PTMC NYHA class for heart failure was found to be negatively correlated to post PTMC net atrioventricular compliance $(\mathrm{r}=-0.712, \mathrm{p}<$ $0.001)$.

\section{Study limitations}

Only in hospital observations were done, no long term outcome was observed.

\section{Conclusion}

It was found from the study that successful PTMC is associated with immediate significant improvement in net atrioventricular compliance. Net atrioventricular compliance is inversely correlated with NYHA functional status, transmitral pressure gradient, pulmonary artery systolic pressure \& means left atrial pressure following successful PTMC. Post PTMC net atrioventricular compliance is also positively correlated with pre PTMC net atrioventricular compliance. There is no significant correlation between net atrioventricular compliance and index left atrial anteroposterior diameter following successful PTMC.

\section{Recommendations}

Further study using large number of patients and inclusion of control group is needed to confirm our findings and to assess their utility in patients follow up and management. Long term follow up of patients following PTMC will establish the ground for prediction of long term outcome depending on post PTMC net atrioventricular compliance. Studies can be under taken to predict severity of right ventricular dysfunction \& to assess patient's probability to develop left atrial thrombus depending on net atrioventricular compliance.

\section{Conflict of Interest - None.}

\section{References:}

1. Islam AKMM, Azhar MA, Islam MF, Haque MZ, Yasmin L. A clinical study on the pathogenesis of mitral vulvular heart disease. Cardiovasc $j$ 2010;3:11-21.

2. Newby DE, Grubb NR and Bradbury A. Cardiovascular disease. In: Davidsons principles and Practice of Medicine. Eds. Colledge NR, Walker BR, Ralston SH. 21st ed. Churchill livingstone Elesvier, Edinburg, 2010: 613.

3. Carapetis JR, Steer AC, Mulholland EK, Weber M. The global burden of group A streptococcal diseases. Lancet Infectious Disease 2005; 5:685-694.
4. Nobuyoshi M, Arita T, Shirai S, Hamasaki N, Yokoi H, Iwabuchi M, et al. Percutaneous balloon mitral valvuloplasty: A Review. Circulation 2009; 119:211-219.

5. Ahmed J, Zaman MM and Hasan MMM. Prevalence of rheumatic fever and rheumatic heart disease in rural Bangladesh. Tropical Doctor 2005; 35: 160-161.

6. Okubo S, Masuda Y, Kawazoa K. Rheumatic heart diseases in Bangladesh and Japan: Difference of clinical feature including two dimensional echocardiographic finding in proceeding of the Bangladesh - Japan Joint Conference on cardiovascular diseases. JICA, Dhaka, 1984:1-9.

7. Rusted IE, Schefley CH and Edward JE. Studies of the mitral valve: Certain anatomic features of the mitral valve and associated structures in mitral stenosis. Circulation 1956; 14:398.

8. Joseph D, Marco John D. Pulmonary and left atrial hemodynamics in mitral stenosis. Am Heart J 1977;94: 73-80.

9. Klabunde RE. Cardiovascular Physiology Concepts. $2^{\text {nd }}$ edition. Philadelphia: Lippincott Williams \& Wilkins, , 2011, 69-74.

10. Chun-Pen Liu, Chih-Tai Ting. Reduced left ventricular compliance in human mitral stenosis. Circulation 1992; 85: $1447-1456$.

11. Schwammenthal E, Vered Z. Impact of atrioventricular compliance on pulmonary artery pressue In mitral stenosis. Circulation 2000; 102: 2378-2384.

12. Song JK, Kang DH, Lee CW, Lee SG, Cheong SS, Hong MK, et al. Factors determining the exercise capacity in mitral stenosis. Am J Cardiol 1996; 78:10601062.

13. Bonow RO, Carabello BA, Chatterjee K, de Leon AC, Jr Faxon DP, Freed MD, et al. 2008 Focused update incorporated into the ACC/AHA 2006 guidelines for the management of patients with valvular heart disease: a report of the American College of Cardiology/American Heart Association Task Force on Practice Guidelines (Writing Committee to Revise the 1998 Guidelines for the Management of Patients With Valvular Heart Disease): endorsed by the Society of Cardiovascular Anesthesiologists, Society for Cardiovascular Angiography and Interventions, and Society of Thoracic Surgeons. Circulation 2008; 118: 523-661.

14. Corte TJ, McDonagh TA, Wort SJ. Pulmonary hypertension in left heart disease: a review. Int $J$ Cardiol 2012; 156: 253-258.

15. Ha JW, Chung N, Jang Y, Kang WC, Kang SM, Rim SJ, et al. Is the left atrial v. wave the determinant of peak pulmonary artery pressure in patients with pure mitral stenosis? Am J Cardiol 2000; 85:986-991.

16. Pande S, Agarwal SK, Dhir U, Chaudhary A, Kumar S, Agarwal V. Pulmonary arterial hypertension in rheumatic mitral stenosis: does it affect right ventricular function and outcome after mitral valve replacement? Interact Cardiovasc Thorac Surg 2009; 9: 421-425. 
17. Guazzi M, Arena R. Pulmonary hypertension with leftsided heart disease. Nat Rev Cardiol 2010; 7:.648-659.

18. Chandrashekhar Y, Westaby S, Narula J. Mitral stenosis. Lancet 2009; 374 :1271-1283.

19. Braunwald E, Moscovitz HL, Amram SS, Lasser RP, Sapin SO, Himmelstein A, et al. The hemodynamics of the left side of the heart as studied by simultaneous left atrial, left ventricular, and aortic pressures; particular reference to mitral stenosis. Circulation 1955; 12: 69-81.

20. Li M, Déry JP, Dumesnil JG, Boudreault JR, Jobin J, Pibarot P. Usefulness of measuring net atrioventricular compliance by Doppler echocardiography in patients with mitral stenosis. Am J Cardiol 2005; 96:432-435.

21. Flachskampf FA, Weyman AE, Guerrero JL, Thomas JD. Calculation of atrioventricular compliance from the mitral flow profile: analytic and in vitro study. $J A m$ Coll Cardiol 1992; 19: 998-1004.

22. Schwammenthal E, Vered Z, Agranat O, Kaplinsky E, Rabinowitz B, Feinberg MS. Impact of atrioventricular compliance on pulmonary artery pressure in mitral stenosis: an exercise echocardiographic study. Circulation 2000; 02: 2378-2384.

23. Nunes MCP, Hung J, Barbosa MM, Esteves WA, Carvalho VT, Lodi-Junqueira L, et al. Impact of Net Atrioventricular Compliance on Clinical Outcome in Mitral Stenosis. Circ Cardiovasc Imaging 2013; 6: 1001-1008;

24. Nunes MCP, Tan T, Elmariah S, Lago R, Inglessis I, Palacios I, et al. Net Atrioventricular Compliance Is an Independent Predictor of Mortality in Mitral Stenosis. J Am Coll Cardiol 2013; 61(10):

25. Kapoor A, Kumar S, Shukla A, Tewari S, Garg N, Goel $\mathrm{P}$, et al. Determinants of left atrial pressure in rheumatic mitral stenosis: role of left atrial compliance and atrial stiffness. Indian Heart $J$ 2004; 56: 27-31.

26. Mannan MA. Factors influencing mitral valve leaflets excursion after percutaneous trans-venous mitral commissurotomy (MD Thesis). Dhaka: University of Dhaka. 2011: 37-43.
27. Kundu SK, Majumder AAS, Halder D, Chakrovortty SK, Khan MR, Dutta B, et al. Immediate impact of percutaneous transevenous mitral commissurotomy on right ventricular function. Cardiovasc $j$ 2012; 5(1): 3-11.

28. Choudhury A K. Comparison of outcome between percutaneous balloon and surgical close mitral commissurotmy in the context of echocardiographic mitral valve morphology in Bangladesh (MD Thesis). Dhaka: University of Dhaka, 1999: 52.

29. Hasan-Ali H, Shams-Eddin H, Abd-Elsayed AA and Maghraby MH. Echocardiographic assessment of mitral valve morphology after percutaneous transevenous mitral commissurotomy (PTMC). Cardiovascular Ultrasound 2007; 5: 48-56.

30. Harrison JK, Wilson JS, Heame SE and Bashore TM. Complication related to percutaneous transvenous mitral commissurotomy. Cathet Cardiovasc Diagn 1994; 2:.52-60.

31. Kang D, Park S, Song J, Kim H, Hong M, Kim J, et al. Long term clinical and echocardiographic outcome of Percutaneous mitral valvuloplasty, Randomized comparison of Inoue and double balloon techniques. $J$ Am Coll Cardiol 2000; 35:169-175.

32. Illius ZM. Factors determining hospital outcome of perctaneous Transevenous mitral commissurotomy (MD Thesis). Dhaka: University of Dhaka, 2005: 54-58.

33. Rahman MM. Evaluation of factors related to mitral regurgitation during percutaneous transevenous mitral commissurotomy in Bangladesh (MD thesis). Dhaka: University of Dhaka, 2000: 72.

34. Drighil A, Bennis A, Mathewson JW, Lancelotti P and Rocha P. Immediate impact of successful percutaneous mitral valve commissurotomy on right ventricular function. European Journal of Echocardiography 2008; 9(4):.536-551.

35. Bari M A. Comparison of echocardiographic outcome of PTMC done by balloon and metallic valvotome (MD thesis). Dhaka: University of Dhaka, 2000: 66. 